\title{
Predictors for Massive Haemorrhage during Caesarean Delivery Due to Placenta Praevia
}

\author{
Mara Burgers' ${ }^{1}$, Katrien Oude Rengerink ${ }^{1}$, Sanne J. Eschbach ${ }^{2}$, Moira M. Muller ${ }^{3}$, \\ Marielle G. van Pampus', Ben W. J. Mol'5, Irene M. de Graaf ${ }^{1}$ \\ ${ }^{1}$ Department of Obstetrics and Gynaecology, Academic Medical Centre, University of Amsterdam, Amsterdam, \\ The Netherlands \\ ${ }^{2}$ Department of Obstetrics and Gynaecology, Leiden University Medical Center, Leiden, The Netherlands \\ ${ }^{3}$ Department of Obstetrics and Gynaecology, Spaarne Ziekenhuis, Hoofddorp, The Netherlands \\ ${ }^{4}$ Department of Obstetrics and Gynaecology, Onze Lieve Vrouwe Gasthuis, Amsterdam, The Netherlands \\ ${ }^{5}$ Department of Obstetrics and Gynaecology, School of Paediatrics and Reproductive Health, University of \\ Adelaide, Adelaide, Australia \\ Email: maraburgers@hotmail.com
}

Received 6 February 2015; accepted 20 February 2015; published 25 February 2015

Copyright (C) 2015 by authors and Scientific Research Publishing Inc.

This work is licensed under the Creative Commons Attribution International License (CC BY).

http://creativecommons.org/licenses/by/4.0/

(c) (i) Open Access

\section{Abstract}

Objectives: To describe the incidence and predictive factors for massive haemorrhage during a caesarean delivery in women with placenta praevia. Methods: We performed a retrospective cohort study among consecutive women with a placenta praevia planned for a caesarean delivery between 2001-2011 in one academic centre and two teaching hospitals in the Netherlands. Massive haemorrhage was defined as $\geq 1000 \mathrm{~mL}$ blood loss during caesarean section. We used logistic regression analysis to assess which maternal and pregnancy characteristics were predictors for massive haemorrhage during caesarean delivery. Results: Of 54,794 deliveries, we identified 215 $(0.39 \%)$ women with placenta praevia who underwent a Caesarean delivery, of whom 94 (44\%) had massive haemorrhage at or after surgery. After univariable preselection, multivariable logistic regression indicated that higher maternal age (OR 2.09; 95\% CI 1.17 - 3.74), no Caucasian ethnicity (OR 1.73; 95\% CI 0.92 - 3.27), multiple pregnancy (OR 3.92; 95\% CI 0.72 - 21.28), lower systolic blood pressure during pregnancy (OR 1.03; 95\% CI 1.00 - 1.07) and the placenta located at the anterior wall (OR 2.21; 95\% CI 1.20 - 4.04) were independent predictors for massive haemorrhage during a caesarean delivery in women with placenta praevia. Predicted probabilities varied between $22 \%$ and $71 \%$. The model has a reasonable discriminative ability and acceptable calibration. Conclusions: Women with placenta praevia are at high risk for massive haemorrhage during caesarean delivery. The risk profile can be used to identify extreme high-risk women in whom extensive preventive measures are justified. 


\section{Keywords}

\section{Placenta Praevia, Massive Haemorrhage, Caesarean Section, Predictors}

\section{Introduction}

Placenta praevia, defined as a placenta that overlies or is proximate $(\leq 2 \mathrm{~cm})$ to the internal ostium (os) of the cervix, has a prevalence at delivery of $0.3 \%-0.5 \%$ [1] [2]. In the United States, maternal mortality occurs in $0.03 \%$ of the cases [2]. Although the aetiology of placenta praevia is unclear, several risk factors for developing a placenta praevia have been described, such as higher maternal age, multiparity, previous caesarean delivery, previous abortion or miscarriage, smoking and cocaine use during pregnancy [3].

Three small retrospective studies suggest that women in whom the distance between the lower placental edge and the internal cervical os was $>2 \mathrm{~cm}$, a so called low-lying placenta, could safely have a vaginal delivery [4]-[6]. Placenta praevia requires an elective caesarean delivery, as delivery is often complicated by massive haemorrhage [1] [7]. Contractions and cervical effacement and dilatation cause the placenta to separate from the uterine wall and provokes bleeding, which can stimulate contractions [1].

If massive haemorrhage cannot be controlled with conservative methods such as uterotonic agents, then surgical haemostatic sutures, radiologic interventions or eventually an emergency hysterectomy are the only life saving procedures. Recent studies showed that in about $0.5 \%$ of all caesarean deliveries a peripartum hysterectomy is done, of which $32 \%$ is due to an uncontrollable bleeding caused by a placenta praevia [8] [9].

Identifying women at high risk for massive haemorrhage due to placenta praevia can help to select the cases in which extra precautions should be taken, like availability of an intervention radiologist and extra venous access during the caesarean section. Risk factors described in a little number of other studies were higher maternal age, previous caesarean section, anterior location of the placenta, total placenta praevia, no bleeding before the caesarean section and the use of general anaesthetics [10]-[13]. A prediction model has currently not been made. Therefore the aim of this study was to further investigate the incidence and predictive factors and to make a prediction model for massive haemorrhage during a caesarean delivery due to a placenta praevia.

\section{Methods}

\subsection{Design and Participants}

We performed a retrospective cohort study. We included all women diagnosed with placenta praevia who delivered between January $1^{\text {st }} 2001$ and December $31^{\text {st }}$ 2011. The study was performed in one university medical centre (Academic Medical Centre in Amsterdam) and two teaching hospitals (Onze Lieve Vrouwe Gasthuis in Amsterdam and Spaarne Ziekenhuis in Hoofddorp).

Women who delivered with a placenta praevia were selected from the delivery registration at the labour wards. A placenta praevia was defined as a placenta with a maximum distance to the internal os of 20 mm, diagnosed by transabdominal and transvaginal ultrasound. When a placenta praevia was suspected at the routine transabdominal ultrasound at 20 weeks of gestation, a transvaginal ultrasound was planned between 28 and 32 weeks of gestation to confirm the diagnosis, or earlier if clinically indicated. In our study the last transvaginal ultrasound was used to confirm the diagnosis. We excluded women who delivered vaginally and women who delivered $<24$ weeks of gestation. We also excluded women with pre-eclampsia, HELLP syndrome, clotting disorders and women using anti-coagulants, as in these disorders vaginal blood loss might have another cause than solely the placenta praevia.

\subsection{Outcome and Possible Risk Factors}

The outcome, major haemorrhage, was defined as $\geq 1000 \mathrm{~mL}$ blood loss during caesarean section. As potential predictors, based on literature search, we considered general and obstetric history, race, age, smoking during pregnancy, Body Mass Index (BMI), use of medication, highest diastolic (DBP) and systolic blood pressure (SBP) during pregnancy, multiple pregnancy, type of placenta praevia (total, partial of marginal), anterior location of the placenta, gestational age at first bleeding, hospitalization, number of hospitalizations, steroids given 
during pregnancy, tocolytics given during pregnancy, Haemoglobin (Hb) level at start pregnancy, iron suppletion or transfusions given during pregnancy, Hb before delivery and gestational age at delivery.

We also determined which type of anaesthesia was used during the delivery. We believe the choice for loco regional or general anaesthesia is often based on clinical judgement and the degree of emergency rather than it being an independent predictor for massive haemorrhage. Because of the risk of confounding by indication this variable was not included in our further analysis. All data were extracted from the electronic patient charts.

\subsection{Analysis}

We used univariable and multivariable logistic regression analysis to assess whether in women with placenta praevia the potential predictors (mentioned above) were associated with major haemorrhage during caesarean delivery.

Factors with a p-value $<0.20$ in the univariable analysis were included in a multivariable model. A multivariable model to identify independent predictors for massive haemorrhage was constructed using backward stepwise elimination. Variables with a p-value $<0.10$ remained in the final model. The crude and adjusted Odds Ratios (OR) and their 95\% confidence intervals (95\% CI) were computed.

We constructed a Kaplan-Meier curve to display the time to delivery in the non-major haemorrhage and the major haemorrhage group. A log-rank test was used to test for differences between groups.

Predictive accuracy and calibration of the model were compared using a ROC curve and the Hosmer and Lemeshow goodness of fit test, respectively. The calibration plot indicates the range of predictions that are made, as well as their reliability. A strongly increased risk of haemorrhage would indicate that preventive measures would be warranted, whereas in low risk predicted patients this would not be necessary.

For the comparison of the baseline characteristics p-values were calculated using the Chi-square test for differences for categorical variables and a t-test for normally distributed continuous variables. For the logistic regression the Wald test was used. All statistical analyses were performed with SPSS version 19.0 (Chicago, IL, USA).

\section{Results}

Of 54.794 women who delivered during the 10-year study period at the three hospitals, 243 (0.44\%) were diagnosed with placenta praevia. Of these, we excluded 28 women for the following reasons: 13 delivered vaginally, 3 delivered before 24 weeks of gestations, 6 had pre-eclampsia or HELLP syndrome, 3 had clotting disorders and 3 used anti-coagulants. The flow chart is shown in Figure 1.

The 215 patients included in the analysis were divided into two groups, 94 (44\%) with massive haemorrhage during delivery and 121 (56\%) without massive haemorrhage. In the group with massive haemorrhage the mean blood loss was $2066 \mathrm{~mL}$ (median: $1500 \mathrm{~mL}$ ), in the group without massive haemorrhage this was $550 \mathrm{~mL}$ (median: $500 \mathrm{~mL}$ ). In the massive haemorrhage group, 87 (93\%) women underwent one or more interventions to stop the bleeding: haemostatic sutures in 73 women (78\%), one embolization of an artery (1.1\%) was performed by an intervention radiologist, and two (2.1\%) emergency hysterectomies were performed. The emergency hysterectomies were performed for an uncontrollable bleeding and due to a placenta accrete respectively. Moreover, 48 women (42\%) had blood transfusion with on average 2 packed cells (range 0 - 16).

In six cases, the placenta praevia was accompanied by placenta accrete (2.8\%), all identified during the caesarean section, of whom five had massive haemorrhage. Six patients had a previous placenta praevia (2.8\%), of which three had massive haemorrhage.

Figure 2 shows the Kaplan Meier curve comparing the gestational age at delivery for women with and without massive haemorrhage. The median gestational age at delivery was 38 weeks +1 day in the group with and 38 weeks +2 days in the group without massive haemorrhage (p-value for difference 0.78 ).

The univariable association between potential predictors and massive haemorrhage is presented in Table 1. Patients with massive haemorrhage were more often 35 years or older (56\% [53/94] vs. 38\% [46/121], p $\leq 0.01)$, with an ethnicity other than Caucasian (64\% [60/94] vs. 76\% [92/121], p = 0.05) and with a history of uterus myomatosus (6\% [6/94] vs. 3\% [3/121], $\mathrm{p}=0.17)$. The highest systolic and diastolic blood pressures during pregnancy were lower in the group with massive haemorrhage (systolic $120 \pm 11$ vs. $123 \pm 12$, p $=0.03$, and diastolic $72 \pm 9$ vs. $75 \pm 8$, p = 0.04). Patients with the placenta located on the anterior wall had more often massive haemorrhage (45\% [42/94] vs. 26\% [31/121], p $\leq 0.01)$. A singleton pregnancy was more frequent in 


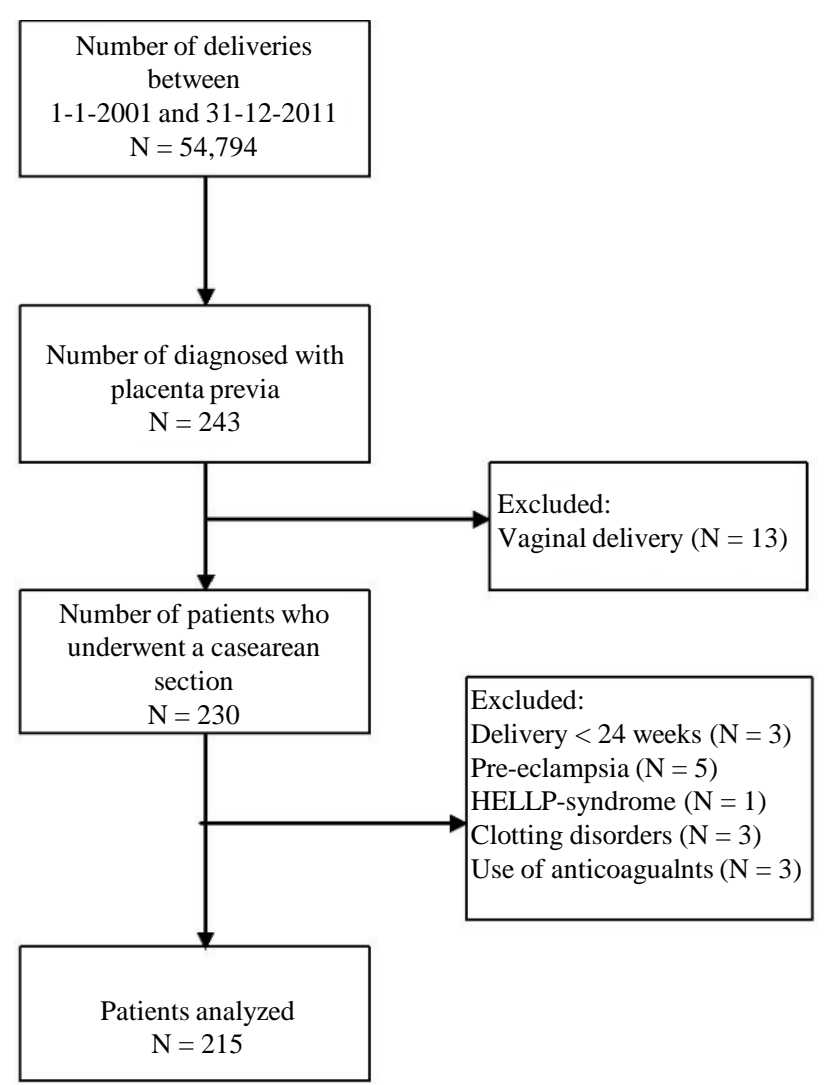

Figure 1. Flowchart of inclusion of patients.

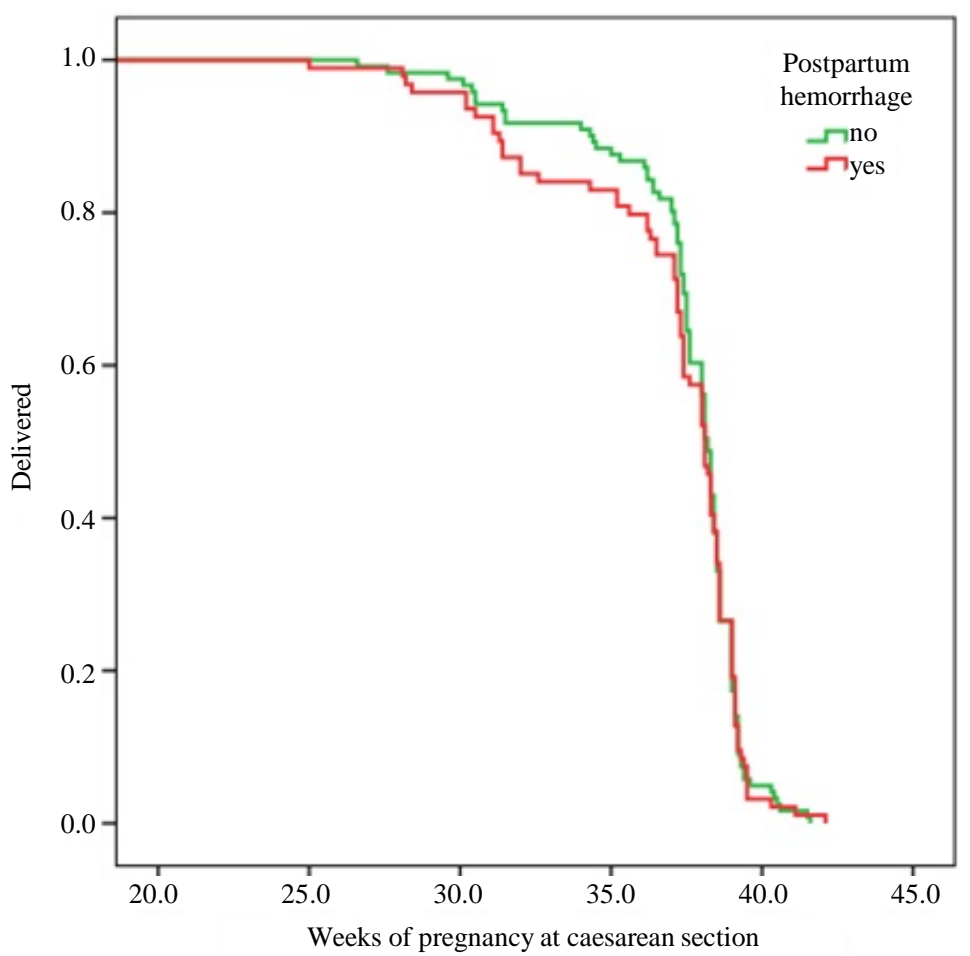

Figure 2. Comparison of the gestational age at delivery for placenta praevia with and without massive haemorrhage. 
Table 1. Univariable analysis of maternal, pregnancy and intrapartum characteristics assosciated with massive haemorrhage.

\begin{tabular}{|c|c|c|c|c|}
\hline $\begin{array}{c}\text { Factors (N, \% unless otherwise } \\
\text { indicated) }\end{array}$ & $\begin{array}{c}\text { Massive haemorrhage, } \\
\qquad \mathrm{N}=94\end{array}$ & $\begin{array}{c}\text { No massive haemorrhage, } \\
\quad \mathrm{N}=121\end{array}$ & $\begin{array}{l}\text { Unadjusted } \mathrm{OR}^{\mathrm{a}} \\
\quad\left(95 \% \mathrm{CI}^{\mathrm{b}}\right)\end{array}$ & p-value \\
\hline \multicolumn{5}{|l|}{ Maternal characteristics } \\
\hline Age $\geq 35$ years & $53(56)$ & $46(38)$ & $2.11(1.22-3.65)$ & $<0.01$ \\
\hline Caucasian & $60(64)$ & $92(76)$ & $0.56(0.31-1.01)$ & 0.05 \\
\hline Smoking during pregnancy & $9(10)$ & $16(15)$ & $0.64(0.28-1.52)$ & 0.31 \\
\hline $\mathrm{BMI}^{* \mathrm{c}}$ & $23.6( \pm 3.7)$ & $24.6( \pm 6.4)$ & $0.96(0.90-1.03)$ & 0.29 \\
\hline Parity, nullipara & 37 (39) & $50(41)$ & $1.09(0.63-1.88)$ & 0.77 \\
\hline Caesarean section in history & $21(22)$ & $20(17)$ & $1.45(0.73-2.87)$ & 0.28 \\
\hline \multicolumn{5}{|l|}{ History of: } \\
\hline Curettage & $25(27)$ & $31(26)$ & $1.05(0.57-1.94)$ & 0.87 \\
\hline Fluxus & $5(5)$ & $7(6)$ & $0.92(0.28-2.98)$ & 0.88 \\
\hline Abdominal surgery & $13(14)$ & $19(16)$ & $0.86(0.40-1.85)$ & 0.70 \\
\hline Intra-uterine surgery & $4(4)$ & $4(3)$ & $1.30(0.32-5.34)$ & 0.72 \\
\hline Uterus myomatosus & $6(6)$ & $3(3)$ & $2.68(0.65-11.02)$ & 0.17 \\
\hline \multicolumn{5}{|l|}{ Pregnancy characteristics } \\
\hline Use of medication & $4(4)$ & $7(6)$ & $0.72(0.21-2.55)$ & 0.62 \\
\hline Highest SBP ${ }^{d}$ during pregnancy ${ }^{*}$ & $120( \pm 11)$ & $123( \pm 12)$ & $0.97(0.95-0.99)$ & 0.03 \\
\hline Highest $\mathrm{DBP}^{\mathrm{e}}$ during pregnancy ${ }^{*}$ & $72( \pm 9)$ & $75( \pm 8)$ & $0.97(0.94-0.99)$ & 0.04 \\
\hline $\mathrm{Hb}^{\mathrm{f}}$ before pregnancy ${ }^{*}$ & $7.6( \pm 0.7)$ & $7.6( \pm 0.8)$ & $0.88(0.61-1.28)$ & 0.51 \\
\hline Singleton pregnancy & $88(94)$ & $119(98)$ & $0.25(0.05-1.25)$ & 0.09 \\
\hline Type of placenta praevia & & & & 0.38 \\
\hline Total & $55(58)$ & $61(50)$ & $1.26(0.69-2.29)$ & \\
\hline Partial & $9(10)$ & $18(15)$ & $0.70(0.28-1.77)$ & \\
\hline Marginal & $30(32)$ & $42(35)$ & $0.88(0.49-1.56)$ & \\
\hline Placenta anterior & $42(45)$ & $31(26)$ & $2.35(1.32-4.17)$ & $<0.01$ \\
\hline First time of bleeding & & & & 0.30 \\
\hline$<24$ weeks & $11(12)$ & $7(6)$ & $1.68(0.57-4.95)$ & \\
\hline 24 - 32 weeks & $29(31)$ & $44(36)$ & $0.71(0.35-1.42)$ & \\
\hline >32 weeks & $26(27)$ & $40(33)$ & $0.69(0.34-1.42)$ & \\
\hline no bleeding & $28(30)$ & $30(25)$ & $1.20(0.65-2.24)$ & \\
\hline Hospitalization & $69(73)$ & $92(76)$ & $0.87(0.47-1.62)$ & 0.66 \\
\hline Number of hospitalizations & & & & 0.81 \\
\hline 0 & $25(27)$ & $29(24)$ & 1 & \\
\hline $1-2$ & $47(50)$ & $60(50)$ & $0.91(0.47-1.75)$ & \\
\hline$>2$ & $22(23)$ & $32(26)$ & $0.79(0.37-1.71)$ & \\
\hline Tocolytic therapy given & $12(13)$ & $14(12)$ & $1.12(0.49-2.55)$ & 0.79 \\
\hline Corticosteroids given & $23(25)$ & $28(23)$ & $1.08(0.57-2.03)$ & 0.82 \\
\hline $\mathrm{Hb}$ before $\mathrm{cs}^{* \mathrm{~g}}$ & $6.9( \pm 0.8)$ & $7.1( \pm 0.7)$ & $0.77(0.54-1.12)$ & 0.17 \\
\hline $\mathrm{Hb}$ medication given & $46(49)$ & $50(41)$ & $1.36(0.79-2.34)$ & 0.27 \\
\hline Cs on planned date & $50(53)$ & $70(58)$ & $0.83(0.48-1.42)$ & 0.50 \\
\hline Gestational age at delivery $^{*}$ & $36.9( \pm 3.2)$ & $37.4( \pm 2.6)$ & $0.94(0.85-1.03)$ & 0.18 \\
\hline \multicolumn{5}{|l|}{ Intrapartum characteristics } \\
\hline Analgesics during $\mathrm{cs}^{* *}$ & & & $2.36(1.31-4.24)$ & $<0.01$ \\
\hline Spinal & $55(59)$ & $93(77)$ & & \\
\hline Narcose & $39(41)$ & $28(23)$ & & \\
\hline
\end{tabular}

*Mean (SD). ${ }^{* *}$ This factor wasn't included in our prediction model, as it is the choice of the clinician based on factors already present, rather than an independent factor. ${ }^{\mathrm{a}}$ Systolic blood pressure; ${ }^{\mathrm{b}}$ Confidence interval; ${ }^{\mathrm{C}}$ Body mass index; ${ }^{\mathrm{d}}$ Systolic blood pressure; ${ }^{\mathrm{e}}$ Diastolic blood pressure; ${ }^{\mathrm{f}} \mathrm{Hae}$ moglobin; ${ }^{\mathrm{g} C a e s a r e a n ~ s e c t i o n . ~}$ 
the group with no massive haemorrhage (94\% [88/94] vs. 98\% [119/121], p = 0.09). The Hb before delivery was lower in the group with massive haemorrhage (6.9 \pm 0.8 vs. $7.1 \pm 0.7, p=0.17)$, and general anaesthesia was more often used $(\mathrm{p} \leq 0.01)$.

Table 2 shows the multivariable analysis. Multivariable logistic regression identified maternal age $\geq 35$ years (OR 2.09; 95\% CI 1.17 - 3.74), no Caucasian ethnicity (OR 1.73; 95\% CI 0.92 - 3.27), a multiple pregnancy (OR 3.92; 95\% CI 0.72 - 21.28), a lower systolic blood pressure during pregnancy (OR 1.03; 95\% CI 1.00 - 1.07) and the placenta located at the anterior wall (OR 2.21; 95\% CI 1.20 - 4.04) as independent predictors for massive haemorrhage. The model had an area under the ROC curve of 0.69 (95\% CI 0.62 - 0.76), indicating reasonable discriminative ability (Figure 3 ).

Figure 4 shows the calibration plot of the observed and predicted risks of massive haemorrhage during caesarean section. The observed and the predicted risks are quite similar, the Hosmer and Lemeshow test assessing calibration showed a good fit (p-value 0.83).

Table 2. Factors associated with massive haemorrhage in multivariable analysis.

\begin{tabular}{cc}
\hline Variable & Adjusted OR (95\% CI) \\
\hline Age $\geq 35$ years & $2.093(1.171-3.741)$ sig 0.013 \\
No Caucasian ethnicity & $1.733(0.919-3.268)$ sig 0.089 \\
Multiple pregnancy & $3.922(0.719-21.276)$ sig 0.114 \\
Lower SBP during pregnancy & $1.031(0.996-1.067)$ sig 0.085 \\
Placenta anterior & $2.205(1.204-4.040)$ sig 0.010
\end{tabular}

${ }^{\mathrm{a}}$ Systolic blood pressure.

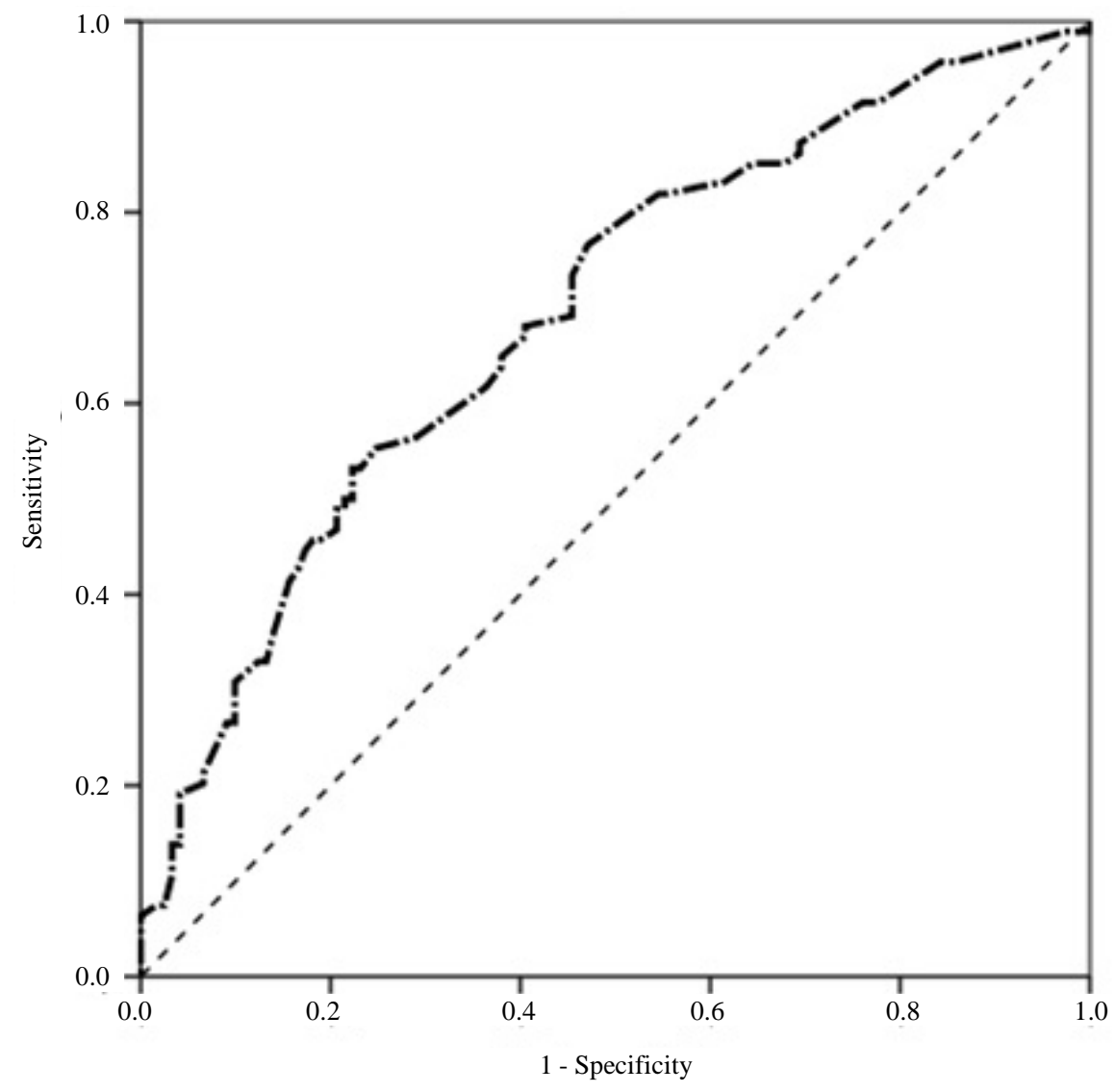

Figure 3. ROC-curve. 


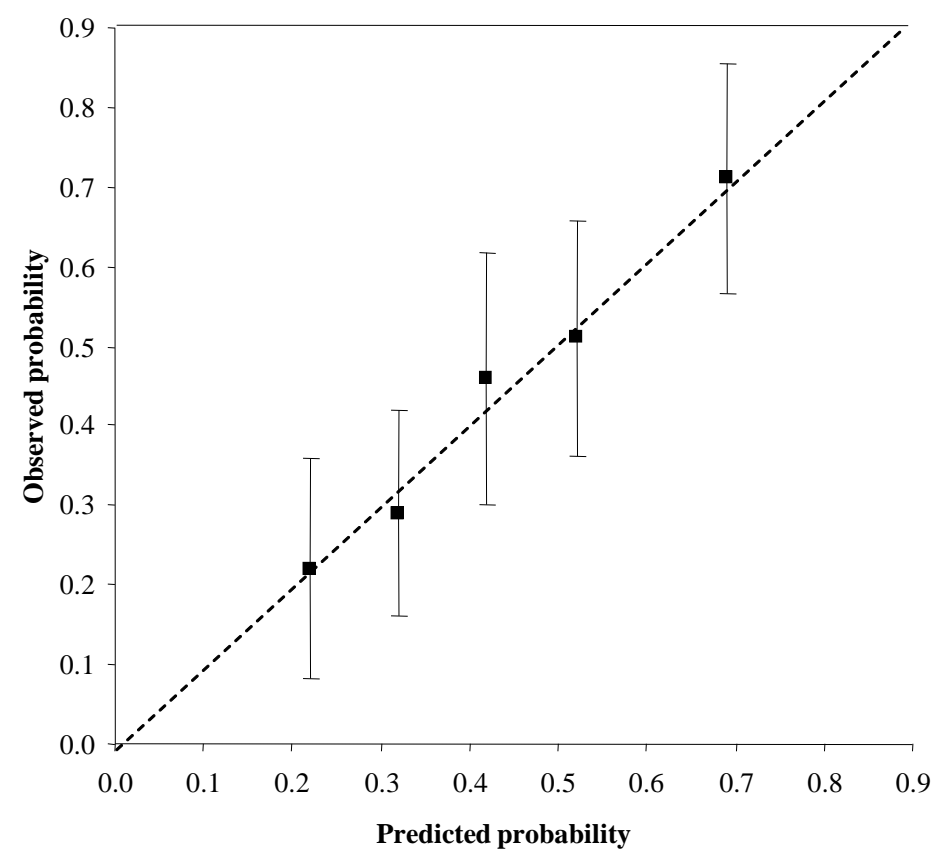

Figure 4. Calibration plot.

\section{Discussion}

Hasegawa et al. also found advanced maternal age and a placenta at the anterior wall as predictors for massive bleeding during the caesarean delivery [11]. They additionally identified a previous caesarean section as a strong risk factor (OR 7.3, 95\% CI 1.9 - 28.2). In our study this was not significant in univariable analysis (OR 1.5, $95 \%$ CI 0.7 - 2.9). Differences in results may be caused by the difference in the definition of major haemorrhage ( $>2500 \mathrm{~mL}$ in the study by Hasegawa $e t$ al. vs. $\geq 1000 \mathrm{~mL}$ in our study).

Jang et al. reported that a placenta at the anterior wall had a higher incidence of massive bleeding compared to a placenta located at the posterior wall (OR 2.9, 95\% CI 1.6 - 5.4) [12]. This finding resembles with our results (OR 2.3; 95\% CI 1.3 - 4.2).

Watanabe et al. reported that patients without blood loss before delivery, bled more than those with blood loss before start of delivery (1563 $\pm 790 \mathrm{ml}$ vs. $1276 \pm 688 \mathrm{ml}, \mathrm{p}=0.02)$ [13]. That finding was not reproduced in our study. We found no difference between the occurrence of no bleeding and massive haemorrhage at delivery (OR 1.20; 95\% CI 0.65 - 2.24). The study population of Watanabe et al. was smaller $(\mathrm{n}=114)$ than ours, with wide ranges of blood loss during/after caesarean section observed in patients both with and without warning bleeding. They suggest further research, to confirm their results.

Our univariable analysis showed that patients who received general anaesthesia during caesarean delivery were more likely to have a massive bleeding (OR 2.36; 95\% CI 1.31 - 4.24, p-value < 0.01). However, we did not include this factor in our prediction model, as the choice of anaesthesia is usually based on clinical factors already present like amount of blood loss at the time of decision, placental localisation and whether or not the delivery is an emergency. Our findings are similar with the findings of Frederiksen et al. [10] and Afolabi et al. [14] who reported that general anaesthesia increased intraoperative blood loss and the need for blood transfusion as compared to regional anaesthesia. This difference can be caused by muscle relaxation and opening of the blood vessels with general anaesthesia. In case of a higher risk for massive haemorrhage, for instance in cases with placenta praevia, general anaesthesia should be avoided.

Strong points of our study are the relatively large sample size. We included all women with a placenta praevia during a 10-year period in three hospitals. Furthermore this study contributes to a better risk estimation en makes the obstetrician more aware of the problems that may be encountered.

Our study has also some weaknesses. The study was retrospective; some cases might have been documented inaccurately and some cases might have been missed due to inaccuracy of diagnosing placenta praevia. However, the retrospective study design allowed us to include a relatively large number of patients with placenta praevia. 
Secondly, the observed and the predicted risks are quite similar, but there is some uncertainty caused by the large confidence intervals (Figure 4). This may lead to situations where a patient at high risk is assigned a low predicted probability, and thus forgoes possible preventive interventions. The first and fifth points are not overlapping indicating a patient in the lowest predicted risk category will have lower observed massive haemorrhage than patients in the highest predicted risk category.

Validation can be done by prospective use of the prediction model. The clinical outcome of two groups, one with and one without use of the model, can be compared. After validation the model may lead to adaption of the policy concerning placenta praevia and as a result reduce the occurrence of massive haemorrhage. We recommend at this stage that women with a placenta praevia and predictors, despite the number, should be treated with caution and precautions must be taken, such as the availability of a multidisciplinary team, notifying an intervention-radiologist, extra venous access and enough blood, medication and uterotonics in the operation room in order to be able to stop the bleeding. A multidisciplinary protocol should be made by gynaecologists, anaesthesiologists, radiologists and coagulation specialists to provide optimal care.

\section{Conclusions}

In this retrospective study the risk of massive blood loss during the caesarean section performed for placenta praevia was high (44\%). Predictors for massive blood loss were a higher maternal age ( $\geq 35$ years) (OR 2.09; 95\% CI 1.17 - 3.74), an ethnicity other than Caucasian (OR 1.73; 95\% CI 0.92 - 3.27), a multiple pregnancy (OR 3.92; 95\% CI 0.72 - 21.28), a lower systolic blood pressure during pregnancy (OR 1.03; 95\% CI 1.00 - 1.07) and the placenta located at the anterior wall (OR 2.21; 95\% CI 1.20 - 4.04).

The risk for massive haemorrhage ranged from $15 \%$ for patients aged under 35 with a Caucasian ethnicity, a singleton pregnancy, the placenta located at the posterior site and a higher systolic blood pressure during pregnancy (123 mmHg \pm 12 ), to $91 \%$ for patients aged over 35 with no Caucasian ethnicity, a multiple pregnancy, the placenta located at the anterior site and a lower systolic blood pressure during pregnancy (120 mmHg \pm 11$)$. Obstetricians should be aware of this high risk.

\section{Conflict of Interest}

All authors declare that they have no conflict of interest.

\section{References}

[1] Oyelese, Y. and Smulian, J.C. (2006) Placenta Previa, Placenta Accreta, and Vasa Previa. Obstetrics \& Gynecology, 107, 927-941. http://dx.doi.org/10.1097/01.AOG.0000207559.15715.98

[2] Iyasu, S., Saftlas, A.K., Rowley, D.L., Koonin, L.M., Lawson, H.W. and Atrash, H.K. (1993) The Epidemiology of Placenta Previa in the United States, 1979 through 1987. American Journal of Obstetrics and Gynecology, 168, 14241429. http://dx.doi.org/10.1016/S0002-9378(11)90776-5

[3] Faiz, A.S. and Ananth, C.V. (2003) Etiology and Risk Factors for Placenta Previa: An Overview and Meta-Analysis of Observational Studies. Journal of Maternal-Fetal and Neonatal Medicine, 13, 175-190. http://dx.doi.org/10.1080/jmf.13.3.175.190

[4] Bhide, A., Prefumo, F., Moore, J., Hollis, B. and Thilaganathan, B. (2003) Placental Edge to Internal os Distance in the Late Third Trimester and Mode of Delivery in Placenta Praevia. BJOG, 110, 860-864. http://dx.doi.org/10.1111/j.1471-0528.2003.02491.x

[5] Dawson, W.B., Dumas, M.D., Romano, W.M., Gagnon, R., Gratton, R.J. and Mowbray, R.D. (1996) Translabial Ultrasonography and Placenta Previa: Does Measurement of the os-Placenta Distance Predict Outcome? Journal of Ultrasound in Medicine, 15, 441-446.

[6] Oppenheimer, L.W., Farine, D., Ritchie, J.W., Lewinsky, R.M., Telford, J. and Fairbanks, L.A. (1991) What Is a Low-Lying Placenta? American Journal of Obstetrics and Gynecology, 165, 1036-1038. http://dx.doi.org/10.1016/0002-9378(91)90465-4

[7] Smith, R.S., Lauria, M.R., Comstock, C.H., Treadwell, M.C., Kirk, J.S., Lee, W., et al. (1997) Transvaginal Ultrasonography for All Placentas That Appear to Be Low-Lying or over the Internal Cervical os. Ultrasound in Obstetrics \& Gynecology, 9, 22-24. http://dx.doi.org/10.1046/j.1469-0705.1997.09010022.X

[8] Shellhaas, C.S., Gilbert, S., Landon, M.B., Varner, M.W., Leveno, K.J., Hauth, J.C., et al. (2009) The Frequency and Complication Rates of Hysterectomy Accompanying Cesarean Delivery. Obstetrics \& Gynecology, 114, $224-229$. 
http://dx.doi.org/10.1097/AOG.0b013e3181ad9442

[9] Whiteman, M.K., Kuklina, E., Hillis, S.D., Jamieson, D.J., Meikle, S.F., Posner, S.F., et al. (2006) Incidence and Determinants of Peripartum Hysterectomy. Obstetrics \& Gynecology, 108, 1486-1492. http://dx.doi.org/10.1097/01.AOG.0000245445.36116.c6

[10] Frederiksen, M.C., Glassenberg, R. and Stika, C.S. (1999) Placenta Previa: A 22-Year Analysis. American Journal of Obstetrics and Gynecology, 180, 1432-1437. http://dx.doi.org/10.1016/S0002-9378(99)70031-1

[11] Hasegawa, J., Matsuoka, R., Ichizuka, K., Mimura, T., Skizawa, A., Farina, A., et al. (2009) Predisposing Factors for Massive Hemorrhage during Cesarean Section in Patients with Placenta Previa. Ultrasound in Obstetrics \& Gynecology, 34, 80-84. http://dx.doi.org/10.1002/uog.6426

[12] Jang, D.G., We, J.S., Shin, J.U., Choi, Y.J., Ko, H.S., Park, I.Y., et al. (2011) Maternal Outcomes According to Placental Position in Placental Precia. International Journal of Medical Sciences, 8, 439-444. http://dx.doi.org/10.7150/ijms.8.439

[13] Waranabe, T. and Matsubara, S. (2010) No Bleeding before, More Bleeding Later: The Relationship between the Presence of Warning Bleeding and the Amount of Bleeding during Caesarean Section in Placenta Praevia. Journal of Obstetrics and Gynaecology, 30, 836. http://dx.doi.org/10.3109/01443615.2010.509827

[14] Afolabi, B.B. and Lesi, F.E.A. (2012) Regional versus General Anaesthesia for Caesarean Section. Cochrane Database of Systematic Reviews, Issue 10, Article ID: CD004350. http://dx.doi.org/10.1002/14651858.CD004350.pub3 
Scientific Research Publishing (SCIRP) is one of the largest Open Access journal publishers. It is currently publishing more than 200 open access, online, peer-reviewed journals covering a wide range of academic disciplines. SCIRP serves the worldwide academic communities and contributes to the progress and application of science with its publication.

Other selected journals from SCIRP are listed as below. Submit your manuscript to us via either submit@scirp.org or Online Submission Portal.
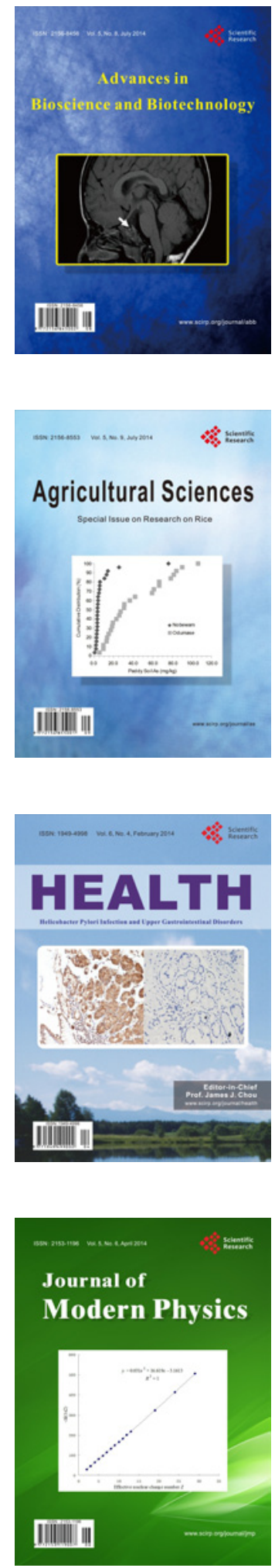
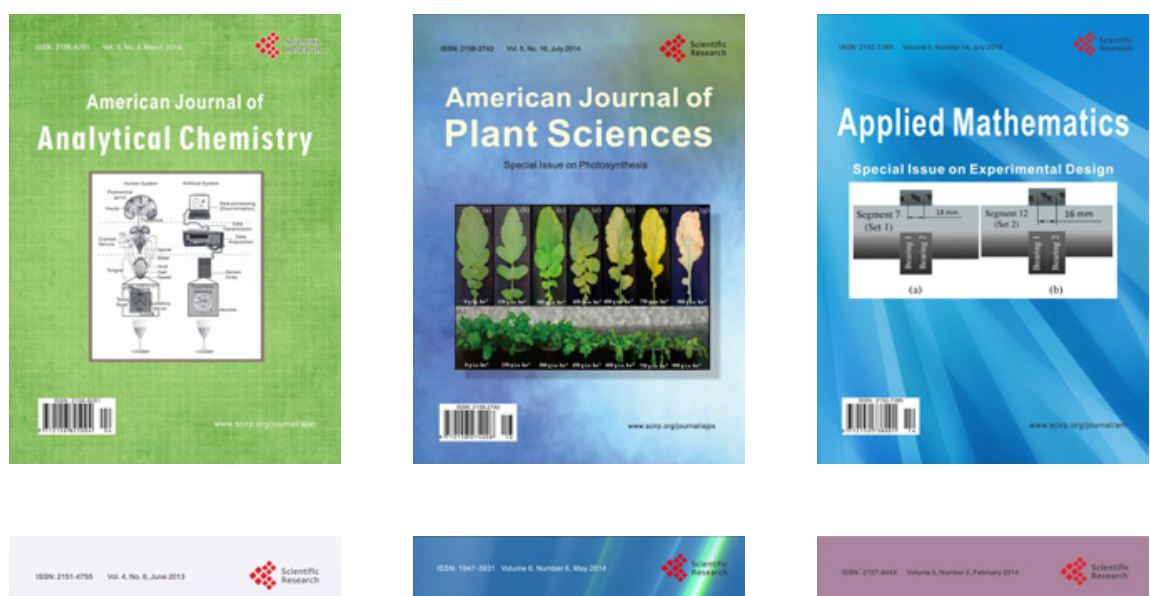

Creative Education
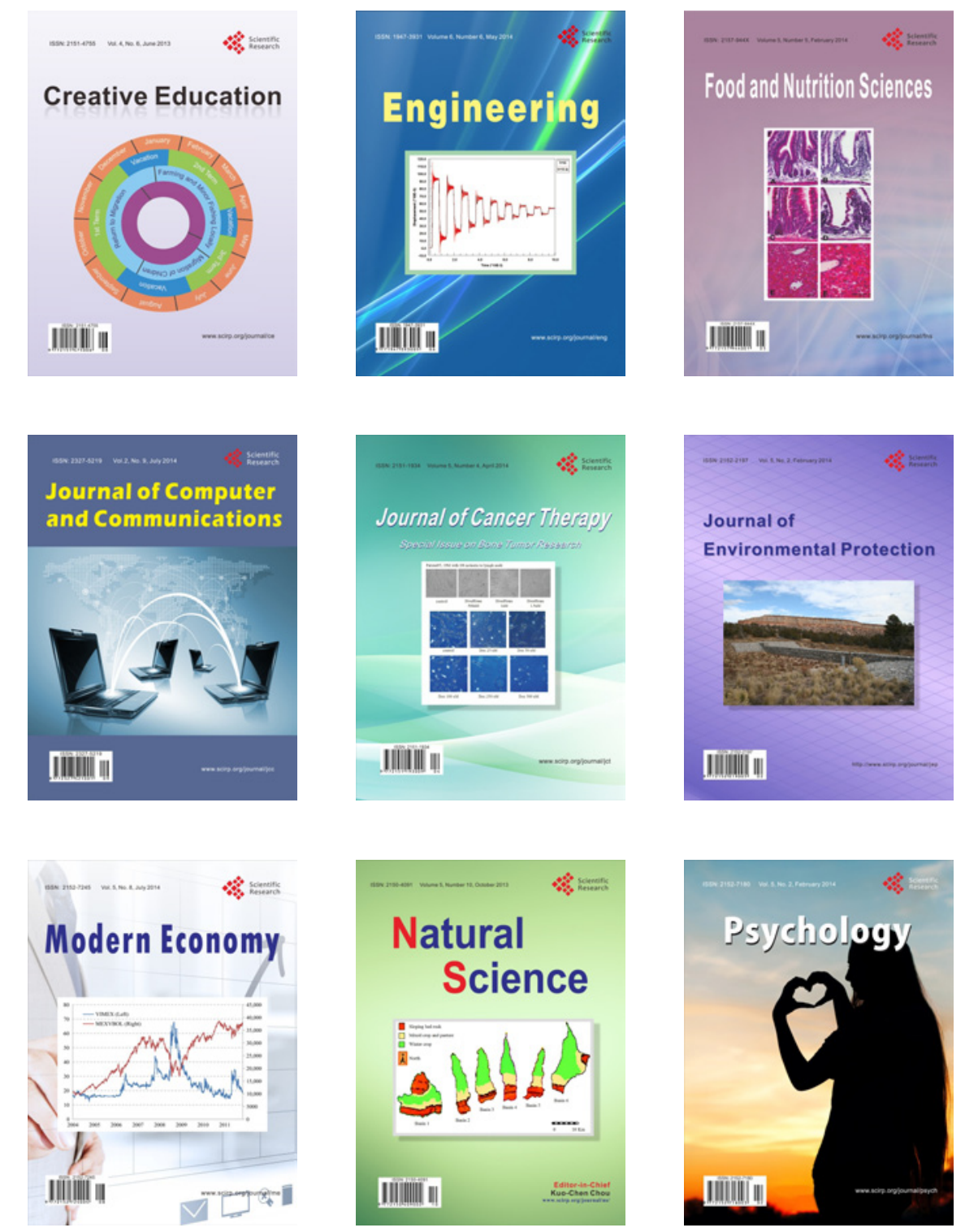